\title{
NILAI TOTAL TAK TERATUR TOTAL DARI GABUNGAN TERPISAH GRAF RODA DAN GRAF BUKU SEGITIGA
}

\author{
R. D. S. Rahangmetan ${ }^{1}$, M. I. Tilukay ${ }^{2}$, F. Y. Rumlawang ${ }^{3}$, M. W. Talakua ${ }^{4}$ \\ 1, 2, 3, 4 Jurusan Matematika FMIPA Universitas Pattimura \\ J1. Ir. M. Putuhena, Kampus Unpatti, Poka-Ambon, Indonesia \\ e-mail: ${ }^{2}$ meilin.tilukay@ fmipa.unpatti.ac.id
}

\begin{abstract}
Abstrak
Pelabelan total tak teratur total yang diperkenalkan oleh Marzuki, dkk merupakan kombinasi dari dua jenis pelabelan tak teratur, yaitu pelabelan total tak teratur titik dan pelabelan total tak teratur titik. Bilangan bulat positif $k$ terkecil sedemikian sehingga suatu graf $G$ memiliki pelabelan $k$-total tak teratur total disebut nilai total tak teratur total dari $G$, dinotasikan dengan $\operatorname{ts}(G)$. Pada makalah ini, nilai total tak teratur total dari gabungan terpisah graf roda dan graf buku segitiga ditentukan.
\end{abstract}

Kata Kunci: Pelabelan total tak teratur sisi, pelabelan total tak teratur titik, pelabelan total tak teratur total.

\section{THE TOTAL IRREGULARITY STRENGTH OF DISJOINT UNION OF WHEEL AND TRIANGULAR BOOK}

\begin{abstract}
A totally irregular total labeling which had been introduced by Marzuki, et.al is a combination of two types of irregular labeling, edge irregular total labeling and vertex irregular total labeling. The minimum integer $k$ for which a graph $G$ has a totally irregular total $k$-labeling is called the total irregularity strength of $G$, denoted by $\operatorname{ts}(G)$. In this paper, we determine the total irregularity strength of disjoint union of wheels and of triangular books.
\end{abstract}

Keywords: edge irregular total labeling, totally irregular total labeling, vertex irregular total labeling.

\section{Pendahuluan}

Diberikan $G$ suatu graf berhingga, sederhana, dan tak berarah, dengan himpunan titik $V$ dan himpunan sisi $E$. Pelabelan graf adalah suatu fungsi yang memetakan elemen-elemen pada graf ke himpunan bilangan (umumnya bilangan bulat positif atau tak negatif).

Pelabelan- $k$ tak teratur (irregular $k$-labeling) dari suatu graf $G$, dengan orde lebih dari 2 , adalah suatu fungsi yang memetakan himpunan sisi $E(G)$ ke himpunan $\{1,2,3, \cdots, k\}$ sedemikian sehingga setiap dua titik yang berbeda di $V(G)$ memiliki bobot yang berbeda. Bilangan bulat positif terkecil $k$ sedemikian sehingga $G$ memiliki suatu pelabelan $-k$ tak teratur disebut nilai ketakteraturan (irregularity strength) dari $G$, dinotasikan dengan $s(G)$.

Selanjutnya, Baca, dkk. [1] memperkenalkan pelabelan tak teratur yang divariasikan berdasarkan domain pelabelan yaitu pelabelan total tak teratur sisi dan pelabelan total tak teratur titik. Misalkan $G=(V, E)$ adalah suatu graf. Pelabelan $-k$ total tak teratur sisi (edge irregular total $k-$ labeling) dari $G$ adalah suatu fungsi $f$ yang memetakan himpunan titik $V(G)$ dan himpunan sisi $E(G)$ ke himpunan $\{1,2,3, \cdots, k\}$ sedemikian sehingga setiap dua sisi yang berbeda di $E(G)$ memiliki bobot yang berbeda. Bobot sisi $x y$ di $E(G)$ terhadap fungsi $f$ adalah $w(x y)=f(x)+f(x y)+f(y)$. Bilangan bulat positif terkecil $k$ sedemikian sehingga $G$ memiliki suatu pelabelan $-k$ total tak teratur sisi disebut nilai total ketakteraturan sisi (total edge irregularity strength) dari $G$, dinotasikan dengan tes $(G)$. Sedangkan pelabelan-k total tak teratur titik (vertex irregular total $k$ - labeling) dari $G$ adalah suatu fungsi $f$ yang memetakan himpunan titik $V(G)$ dan himpunan sisi $E(G)$ ke himpunan $\{1,2,3, \cdots, k\}$ sedemikian sehingga setiap dua titik yang berbeda di $V(G)$ memiliki 
bobot yang berbeda. Bobot titik $x$ di $V(G)$ terhadap fungsi $f$ adalah $w(x)=f(x)+\sum_{x y \in E(G)} f(x y)$. Bilangan bulat positif terkecil $k$ sedemikian sehingga $G$ memiliki suatu pelabelan-k total tak teratur titik disebut nilai total ketakteraturan titik (total vertex irregularity strength) dari $G$, dinotasikan dengan $\operatorname{tvs}(G)$.

Baca dkk. [1] telah memberikan batas bawah dan batas atas nilai total ketakteraturan titik $\operatorname{tvs}(G)$ dan nilai total ketakteraturan sisi tes $(G)$ sebagai berikut.

Teorema A. Untuk setiap graf $G$ dengan $p$ titik dan $q$ sisi, dan derajat minimum $\delta(G)$ serta derajat maksimum $\Delta(G)$

i) $\left\lceil\frac{p+\delta(G)}{\Delta(G)+1}\right\rceil \leq \operatorname{tvs}(G) \leq p+\Delta(G)-2 \delta(G)+1$;

ii) $\left\lceil\frac{|E(G)|+2}{3}\right\rceil \leq \operatorname{tes}(G) \leq|E(G)|$.

Selanjutnya, Wijaya dan Slamin [2] telah menentukan nilai tes dan tvs untuk graf roda dengan $n+1$ titik, $n \geq 3$, yaitu tes $\left(W_{n}\right)=\left\lceil\frac{2 n+2}{3}\right\rceil$ dan $\operatorname{tvs}\left(W_{n}\right)=\left\lceil\frac{n+3}{4}\right\rceil$. Nurdin, dkk. [3] telah menentukan nilai tes untuk graf hasil korona graf lintasan dengan beberapa graf tertentu. Nilai tes dan tvs dari graf-graf lainnya dapat dilihat dalam hasil survey pelabelan graf oleh Galian [4].

Marzuki, Salman, dan Miller [5] menggabungkan ide kedua pelabelan total tersebut dengan memperkenalkan pelabelan total tak teratur titik dan sisi. Misalkan $G=(V, E)$ adalah suatu graf. Pelabelan $-k$ total tak teratur titik dan sisi (totally irregular total k-labeling) pada $G$ didefinisikan sebagai suatu fungsi $f$ yang memetakan himpunan titik $V(G)$ dan himpunan sisi $E(G)$ ke himpunan $\{1,2,3, \cdots, k\}$ sedemikian sehingga setiap dua titik yang berbeda di $V(G)$ memiliki bobot yang berbeda dan setiap dua sisi yang berbeda di $E(G)$ memiliki bobot yang berbeda juga. Bilangan bulat positif terkecil $k$ sedemikian sehingga suatu graf $G$ memiliki pelabelan- $k$ total tak teratur titik dan sisi disebut nilai ketakteraturan total (total irregularity strength) dari $G$, dinotasikan dengan $t s(G)$. Marzuki, Salman, dan Miller [5] telah memberikan batas atas dari ts $(G)$.

Teorema B. Untuk sebarang graf $G$,

$$
t s(G) \geq \max \{\operatorname{tes}(G), \operatorname{tvs}(G)\} .
$$

Untuk beberapa jenis graf, seperti graf lintasan (path), graf lingkaran (cycle) [5] graf hasil kali kartesius dari beberapa graf [6], graf kipas, graf roda, graf buku segitiga, dan graf persahabatan [7] juga telah ditentukan nilai total tak teratur totalnya. Dalam [7], Tilukay, dkk telah menentukan nilat total ketakteraturan total dari graf kipas, graf roda, graf buku segitiga, dan graf persahabatan, sebagai berikut.

Teorema C. Untuk setiap bilangan bulat $n \geq 3$ dan $W_{n}$ merupakan graf roda dengan $n+1$ titik dan $2 n$ sisi. Maka

$$
t s\left(W_{n}\right)=\left\lceil\frac{2 n+2}{3}\right\rceil .
$$

Teorema D. Diberikan $n \geq 3$ dan $P_{1} \odot S_{n}$ merupakan graf buku segitiga dengan $n$ segitiga dengan $n+1$ titik dan $2 n-1$ sisi. Maka

$$
t s\left(P_{1} \odot S_{n}\right)=\left\lceil\frac{2 n+3}{3}\right\rceil .
$$

Dalam penelitian ini, akan dikaji pelabelan total tak teratur total dari gabungan terpisah graf roda dan graf buku segitiga. Permasalahan dibatasi pada gabungan terpisah graf roda $\left(m W_{n}\right)$, untuk $n \equiv 0 \bmod 3$ dan graf buku segitiga $\left(m\left(P_{1} \odot S_{n}\right)\right)$, untuk $n \equiv 1 \bmod 3$. 


\section{Hasil dan Pembahasan}

Diberikan $W_{n}$ suatu graf roda dengan $n+1$ titik dan $2 n$ sisi. Graf $m W_{n}$ adalah suatu graf yang diperoleh dengan menggabungkan $m$ graf roda dengan karakteristik yang sama tanpa menghubungkan sebarang pasang titik atau sisi dari dua graf roda berbeda. Graf $m W_{n}$ disebut juga gabungan terpisah (disjoint union) $m$ graf roda dan memiliki $m(n+1)$ titik dan $2 m n$ sisi.

Tilukay dkk. telah menentukan nilai total tak teratur total dari graf roda $W_{n}$. Dengan memeriksa sifat pelabelan total tak teratur total pada $W_{n}$, dapat diketahui bahwa untuk $n \equiv 0 \bmod 3$, bobot sisi $w\left(v_{n-n} v_{n}\right)=n+3+n-1=2 n+2$ merupakan bobot sisi terbesar. Akibatnya dapat dilakukan pelabelan dengan pola serupa pada $m$-kopi graf $W_{n}, n \equiv 0 \bmod 3$, dengan nilai label yang ditingkatkan berdasarkan kardinalitas himpunan sisi $E\left(m W_{n}\right)$.

Hal ini disajikan dalam lema berikut:

Lema 1. Misalkan $n \geq 3$ dan $m \geq 2$. Jika $m W_{n}$ adalah $m$-kopi graf roda dengan $n$ titik, dimana $n \equiv 0 \bmod 3$, maka

$$
t s\left(m W_{n}\right)=\frac{2 m n}{3}+1 .
$$

Bukti. Diketahui $\left|V\left(m W_{n}\right)\right|=m(n+1)$ dan $\left|E\left(m W_{n}\right)\right|=2 m n$. Berdasarkan Teorema A dan B, $\operatorname{tes}\left(m W_{n}\right) \geq\left\lceil\frac{\left|E\left(m W_{n}\right)+2\right|}{3}\right\rceil=\left\lceil\frac{2 m n+2}{3}\right\rceil$ sedangkan $\operatorname{tvs}\left(m W_{n}\right) \geq\left\lceil\frac{\left|V\left(m W_{n}\right)+3\right|}{4}\right\rceil=\left\lceil\frac{m n+m+3}{4}\right\rceil$ maka telah diperoleh batas bawah nilai $t s\left(m\left(W_{n}\right)\right)$.

Untuk membuktikan bahwa $\left\lceil\frac{2 m n+2}{3}\right\rceil$ merupakan batas atas $\left(m\left(W_{n}\right)\right)$, konstruksikan pelabelan total tak teratur total $f: V \cup E \rightarrow\left\{1,2, \cdots, t_{m}\right\}$ sebagai berikut:

Misalkan $V\left(m W_{n}\right)=\left\{u_{i} \mid 1 \leq i \leq m\right\} \cup\left\{v_{i}^{j} \mid 1 \leq i \leq m, 1 \leq j \leq n\right\}$ dan

$E\left(m W_{n}\right)=\left\{u_{i} v_{i}^{j}, v_{i}^{j} v_{i}^{j+1}, v_{i}^{n} v_{i}^{1} \mid 1 \leq i \leq m, 1 \leq j \leq n\right\}$.

Misalkan $t_{i}=\left\lceil\frac{2 n i+2}{3}\right\rceil=\frac{2 n i}{3}+1$ dan $t_{0}=1$, diperoleh pelabelan titik sebagai berikut:

$f\left(u_{i}\right)=t_{i}-1, \quad 1 \leq i \leq m$;

$f\left(v_{i}^{j}\right)=\left\{\begin{aligned} t_{i-1}+\left\lceil\frac{j}{2}\right]-1, & 1 \leq i \leq m, 1 \leq j \leq t_{1}-1 ; \\ t_{i}, & 1 \leq i \leq m, t_{1} \leq j \leq n\end{aligned}\right.$

dan pelabelan sisi sebagai berikut:

$f\left(u_{i} v_{i}^{j}\right)=\left\{\begin{array}{cl}t_{i-1}+\left\lceil\frac{j+1}{2}\right\rceil-1, & 1 \leq i \leq m, 1 \leq j \leq t_{1}-1 ; \\ t_{i-1}+j-t_{1}+2, & 1 \leq i \leq m, t_{1} \leq j \leq n .\end{array}\right.$

$f\left(v_{i}^{n} v_{i}^{1}\right)=t_{i}-1, \quad 1 \leq i \leq m$.

$f\left(v_{i}^{j} v_{i}^{j+1}\right)= \begin{cases}t_{i-1}, & 1 \leq i \leq m, 1 \leq j \leq t_{1}-2 ; \\ t_{i-1}+\left\lceil\frac{t_{1}}{2}\right\rceil, & 1 \leq i \leq m, j=t_{1}-1 ; \\ t_{i-1}+n-2 t_{1}+j+2, & 1 \leq i \leq m, t_{1} \leq j \leq n-1 .\end{cases}$

Dapat dilihat bahwa label terbesar adalah $f\left(V_{m}^{n}\right)=t_{m}$.

Selanjutnya dengan memberikan label titik-titik dan sisi-sisi graf $m W_{n}$ dengan bilangan terbesar $t_{m}$, akan ditunjukkan bahwa bobot setiap titik dan setiap sisi pada $m W_{n}$ berbeda.

a. Bobot Sisi

$$
\begin{aligned}
w\left(u_{i} v_{i}^{j}\right) & =f\left(u_{i}\right)+f\left(u_{i} v_{i}^{j}\right)+f\left(v_{i}^{j}\right) ; \\
& = \begin{cases}t_{i}+2 t_{i-1}+\left\lceil\frac{j+1}{2}\right\rceil+\left\lceil\frac{j}{2}\right\rceil-3, & 1 \leq i \leq m, 1 \leq j \leq t_{1}-1 ; \\
2 t_{i}+t_{i-1}-t_{1}+j+1, & 1 \leq i \leq m, \quad t_{1} \leq j \leq n .\end{cases}
\end{aligned}
$$




$$
\begin{aligned}
w\left(v_{i}^{j} v_{i}^{j+1}\right)= & f\left(v_{i}^{j}\right)+f\left(v_{i}^{j} v_{i}^{j+1}\right)+f\left(v_{i}^{j+1}\right) ; \\
= & \begin{cases}\left(t_{i-1}+\left\lceil\frac{j}{2}\right]-1\right)+t_{i-1}+\left(t_{i-1}+\left\lceil\frac{j+1}{2}\right\rceil-1\right), 1 \leq i \leq m, 1 \leq j \leq t_{1}-2 ; \\
\left(t_{i-1}+\left\lceil\frac{j}{2}\right\rceil-1\right)+\left(t_{i-1}+\left\lceil\frac{t_{1}}{2}\right\rceil\right)+t_{i}, & 1 \leq i \leq m, j=t_{1}-1 ; \\
t_{i}+t_{i-1}+n-2 t_{1}+j+2+t_{i}, & 1 \leq i \leq m, \quad t_{1} \leq j \leq n-1 .\end{cases} \\
w\left(v_{i}^{n} v_{i}^{1}\right)= & f\left(v_{i}^{n}\right)+f\left(v_{i}^{n} v_{i}^{1}\right)+f\left(v_{i}^{1}\right) ; \\
= & 2 t_{i}+t_{i-1}+\left\lceil\frac{j}{2}\right\rceil-2, \quad 1 \leq i \leq m, j=1 .
\end{aligned}
$$

b. Bobot Titik

$$
\begin{aligned}
w\left(u_{i}\right)= & f\left(u_{i}\right)+\sum_{j=1}^{n} f\left(u_{i} v_{i}^{j}\right) \\
= & t_{i}-1+\left(t_{1}-1\right)\left(t_{i-1}+\left\lceil\frac{j+1}{2}\right\rceil-1\right)+\left(n-t_{1}+1\right)\left(t_{i-1}+j-t_{1}+2\right) ; \\
w\left(v_{i}^{1}\right)= & f\left(v_{i}^{1}\right)+f\left(v_{i}^{1} v_{i}^{2}\right)+f\left(v_{i}^{n} v_{i}^{1}\right)+f\left(v_{i} v_{i}^{1}\right) \\
= & 3 t_{i-1}+t_{i}+\left\lceil\frac{j}{2}\right\rceil+\left\lceil\frac{j+1}{2}\right\rceil-3 . \\
w\left(v_{i}^{j}\right)= & f\left(v_{i}^{j}\right)+f\left(v_{i}^{j-1} v_{i}^{j}\right)+f\left(v_{i}^{j} v_{i}^{j+1}\right)+f\left(v_{i} v_{i}^{j}\right) \\
= & t_{i}+3 t_{i-1}+\left\lceil\frac{j+1}{2}\right\rceil+\left\lceil\frac{j}{2}\right\rceil-3, \quad 1 \leq i \leq m, 2 \leq j \leq t_{1}-2 . \\
w\left(v_{i}^{t_{1}-1}\right)= & f\left(v_{i}^{t_{1}-1}\right)+f\left(v_{i}^{t_{1}-2} v_{i}^{t_{1}-1}\right)+f\left(v_{i}^{t_{1}-1} v_{i}^{t_{1}}\right)+f\left(u_{i} v_{i}^{t_{1}-1}\right) \\
& =t_{i}+3 t_{i-1}+\left\lceil\frac{j+1}{2}\right\rceil+\left\lceil\frac{1}{2}\right\rceil+\left\lceil\frac{t_{1}}{2}\right\rceil-3, \quad 1 \leq i \leq m, j=t_{1}-1 . \\
w\left(v_{i}^{t_{1}}\right)= & f\left(v_{i}^{t_{1}}\right)+f\left(v_{i}^{t_{1}-1} v_{i}^{t_{1}}\right)+f\left(v_{i}^{t_{1}} v_{i}^{t_{1}+1}\right)+f\left(u_{i} v_{i}^{t_{1}}\right) ; \\
= & 3 t_{i-1}-2 t_{i}+2 j+\left\lceil\frac{t_{1}}{2}\right\rceil+n+4, \quad 1 \leq i \leq m, j=t_{1} . \\
w\left(v_{i}^{j}\right)= & f\left(v_{i}^{j}\right)+f\left(v_{i}^{j-1} v_{i}^{j}\right)+f\left(v_{i}^{j} v_{i}^{j+1}\right)+f\left(u_{i} v_{i}^{j}\right), t+1 \leq j \leq n-1 ; \\
= & 3 t_{i-1}-3 t_{i}-t_{1}+2 n+3 j+6 . \\
w\left(v_{i}^{n}\right)= & f\left(v_{i}^{n}\right)+f\left(v_{i}^{n-1} v_{i}^{n}\right)+f\left(v_{i}^{n} v_{i}^{1}\right)+f\left(u_{i} v_{i}^{n}\right) ; \\
= & 2 t_{i-1}+2 j+n-3-t_{1} .
\end{aligned}
$$

Dapat diperiksa bahwa berdasarkan (i), bobot sisi-sisi membentuk barisan $3,4, \cdots, 2 m n+2$, dan bobot setiap titik berbeda, yaitu $w\left(u_{i}\right)>w\left(v_{i}^{n}\right) ; \quad w\left(v_{i}^{j}\right)>w\left(v_{i}^{j-1}\right), 2 \leq j \leq n$; dan $w\left(u_{i}\right)>w\left(u_{i-1}\right), 2 \leq i \leq m$. Dengan demikian, diperoleh bahwa $t s\left(m W_{n}\right)=\left\lceil\frac{2 m n+2}{3}\right\rceil$.

Selanjutnya dengan mengacu pada sifat pelabelan total tak teratur total pada graf buku segitiga, $P_{1} \odot S_{n}$ dapat di lihat bahwa untuk $n \equiv 1 \bmod 3$, nilai $t s\left(P_{1} \odot S_{n}\right)=\left\lceil\frac{2 n+3}{3}\right\rceil$. Diperoleh bobot sisi $W\left(v v_{n}\right)=2 n+3 \equiv 2 \bmod 3$.

Hal ini mengakibatkan dapat dilakukan pelabelan dengan pola yang serupa pada $m$-kopi graf buku segitiga. Pada Lema 2, akan ditentukan nilai total tak teratur total dari $m$-kopi graf buku segitiga.

Lema 2. Misalkan $n \geq 3$ dan $G \cong\left(P_{1} \odot S_{n}\right)$ adalah graf buku segitiga dengan $n$ halaman segitiga. Untuk $n \equiv 1 \bmod 3$ dan $\mathrm{m} \geq 1$,

$$
\text { ts }(m G)=\left\lceil\frac{m(2 n+1)+2}{3}\right\rceil \text {. }
$$

Bukti. Karena $V(m G)=m(n+2)$ dan $E(m G)=m(2 n+1)$, maka berdasarkan Teorema B dan C, diperoleh $\quad t s(m G) \geq\left\lceil\frac{m(2 n+1)+2}{3}\right\rceil$. Misalkan $t_{i}=\left\lceil\frac{i(2 n+1)+2}{3}\right\rceil$, akan ditunjukan bahwa 
ts $(m G) \leq\left\lceil\frac{m(2 n+1)+2}{3}\right\rceil$. Untuk membuktikannya, konstruksikan suatu pelabelan total tak teratur $f: V \cup E \rightarrow\left\{1,2, \ldots,\left\lceil\frac{m(2 n+1)+2}{3}\right]\right\}$.

Misalkan $\quad V(m G)=\left\{x_{i} \mid 1 \leq i \leq m\right\} \cup\left\{y_{i} \mid 1 \leq i \leq m\right\} \cup\left\{v_{i}^{j} \mid 1 \leq i \leq m, 1 \leq j \leq n\right\} \quad$ dan $E(m G)=\left\{x_{i} y_{i}, x_{i} v_{i}^{j}, y_{i} v_{i}^{j} \mid 1 \leq i \leq m, 1 \leq j \leq n\right\}$.

Untuk himpunan titik-titik $V(m G)$, definisikan:

$f\left(x_{i}\right)=t_{i-1}, 1 \leq i \leq m$;

$f\left(y_{i}\right)=t_{i-1}, 1 \leq i \leq m$;

$f\left(v_{i}^{j}\right)= \begin{cases}t_{i-1}+j-1, & 1 \leq i \leq m, 1 \leq j \leq t_{1} ; \\ t_{i}, & 1 \leq i \leq m, t_{1}+1 \leq j \leq n .\end{cases}$

Untuk himpunan sisi-sisi $E(m G)$, definisikan:

$f\left(x_{i} y_{i}\right)=t_{i}, \quad 1 \leq i \leq m$;

$f\left(x_{i} v_{i}^{j}\right)=\left\{\begin{array}{cl}t_{i-1}, & 1 \leq i \leq m, 1 \leq j \leq t_{1} ; \\ j-t_{1}+t_{i-1}, & 1 \leq i \leq m, t_{1}+1 \leq j \leq n ;\end{array}\right.$

$f\left(y_{i} v_{i}^{j}\right)=\left\{\begin{array}{lr}n-t_{1}+t_{i-1}+1, & 1 \leq i \leq m, 1 \leq j \leq \frac{t_{1}}{2} ; \\ n-t_{1}+t_{i-1}+2, & 1 \leq i \leq m, \frac{t_{1}}{2}+1 \leq j \leq t_{1} \\ n-2 t_{1}+t_{i-1}+j+2, & 1 \leq i \leq m, t_{i}+1 \leq j \leq n .\end{array}\right.$

Dapat dilihat bahwa label terbesar yang digunakan adalah $t_{m}$, yaitu pada

$f\left(y_{m}\right)=t_{m}$;

$f\left(x_{m} y_{m}\right)=t_{m}$

$f\left(v_{m}^{j}\right)=t_{m}, t_{1}+1 \leq j \leq n$.

Selanjutnya, dapat diperoleh bobot setiap titik dan sisi sebagai berikut:

a. Bobot titik-titik:

$$
\begin{aligned}
w\left(x_{i}\right) & =f\left(x_{i}\right)+f\left(x_{i} y_{i}\right)+\sum_{j=1}^{n} f\left(x_{i} v_{1}^{j}\right) \\
& =t_{i-1}\left(t_{1}+1\right)+t_{i}+\left(\frac{n-t_{1}+1+2 t_{i-1}}{2}\right)\left(n-t_{1}\right) \\
w\left(y_{i}\right) \quad & f\left(y_{i}\right)+f\left(x_{i} y_{i}\right)+\sum_{j=1}^{n} f\left(y_{i} v_{i}^{j}\right) \\
& =2 t_{i}-t_{1}(3 n+1)+n t_{i-1}+\frac{5 n+3 n^{2}}{2}+\frac{9 t_{1}^{2}}{4} \\
w\left(v_{i}^{j}\right) \quad & f\left(x_{i} v_{i}^{j}\right)+f\left(y_{i} v_{i}^{j}\right)+f\left(v_{i}^{j}\right) \\
& = \begin{cases}3 t_{i-1}-t_{1}+n+j, & 1 \leq i \leq m, 1 \leq j \leq \frac{t_{1}}{2} ; \\
3 t_{i-1}-t_{1}+n+j+1, & 1 \leq i \leq m, \quad \frac{t_{1}}{2}+1 \leq j \leq t_{1} ; \\
t_{i}+2 t_{i-1}-3 t_{1}+n+2 j+2, & 1 \leq i \leq m, t_{1}+1 \leq j \leq n .\end{cases}
\end{aligned}
$$

Dapat diperiksa bahwa bobot setiap titik berbeda.

b. Bobot sisi-sisi:

$$
\begin{array}{rlrl}
w\left(x_{i} y_{i}\right) & =f\left(x_{i}\right)+f\left(y_{i}\right)+f\left(x_{i} y_{i}\right)=t_{i-1}+2 t_{i} . \\
w\left(x_{i} v_{i}^{j}\right) & =f\left(x_{i}\right)+f\left(y_{i}\right)+f\left(x_{i} v_{i}^{j}\right) ; & \\
& =\left\{\begin{array}{cc}
3 t_{i-1}+j-1, & 1 \leq i \leq m, 1 \leq j \leq t_{1} ; \\
t_{i}+2 t_{i-1}-t_{1}+j, & 1 \leq i \leq m, t_{1}+1 \leq j \leq n .
\end{array}\right.
\end{array}
$$




$$
\begin{aligned}
w\left(y_{i} v_{i}^{j}\right)= & f\left(y_{i}\right)+f\left(v_{i}^{j}\right)+f\left(y_{i} v_{i}^{j}\right) ; \\
= & \left\{\begin{array}{l}
t_{i}+2 t_{i-1}-t_{1}+n+j, \quad 1 \leq i \leq m, \quad 1 \leq j \leq \frac{t_{1}}{2} \\
t_{i}+2 t_{i-1}-t_{1}+n+j+1, \quad 1 \leq i \leq m, \quad \frac{t_{1}}{2}+1 \leq j \leq t_{1} ; \\
2 t_{i}+t_{i-1}-2 t_{1}+n+j+2, \quad 1 \leq i \leq m, t_{1}+1 \leq j \leq n .
\end{array}\right.
\end{aligned}
$$

Diperoleh,

a. $\left\{w\left(x_{i} v_{i}^{j}\right) \mid 1 \leq i \leq m, 1 \leq j \leq n\right\}=\left\{3,4, \cdots, t_{i}+2 t_{i-1}-t_{1}+n \mid 1 \leq j \leq m\right\}$

b. $\left\{w\left(y_{i} v_{i}^{j}\right) \mid 1 \leq i \leq m, 1 \leq j \leq \frac{t_{1}}{2}\right\}=\left\{t_{i}+2 t_{i-1}-t_{1}+n+1, t_{i}+2 t_{i-1}-t_{1}+n+2, \cdots, 2 t_{i}+t_{i-1}-\right.$ $1 \mid 1 \leq i \leq m\}$

c. $\left\{w\left(x_{i} y_{i}\right) \mid 1 \leq i \leq m\right\}=\left\{t_{i-1}+2 t_{i} \mid 1 \leq i \leq m\right\}$

d. $\left\{w\left(y_{i} v_{i}^{j}\right) \mid 1 \leq i \leq m, \frac{t_{1}}{2}+1 \leq j \leq t_{1}\right\}=\left\{t_{i}+2 t_{i-1}-\frac{t_{1}}{2}+n+2, t_{i}+2 t_{i-1}-\frac{t_{1}}{2}+n+3, \cdots, t_{i}+\right.$

$$
\left.2 t_{i-1}+n+1\right\} \text {. }
$$

e. $\left\{w\left(y_{i} v_{i}^{j}\right) \mid 1 \leq i \leq t_{1}+1 \leq j \leq n\right\}=\left\{2 t_{i}+t_{i-1}-2 t_{1}+n+3,2 t_{i}+t_{i-1}-2 t_{1}+n+4, \cdots, 2 t_{i}+\right.$ $\left.t_{i-1}-2 t_{1}+2 n+2\right\}$.

Dapat diperiksa bahwa himpunan bobot sisi-sisi adalah $\{3,4, \cdots, m(2 n+1)+2\}$.

Dengan demikian, dapat diperiksa bahwa bobot setiap pasang titik maupun setiap pasang sisi berbeda.

Jadi, fungsi $f$ adalah pelabelan total tak teratur titik dan sisi, sehingga $t s(m G)=\left\lceil\frac{m(2 n+1)+2}{3}\right\rceil$.

\section{Kesimpulan}

Berdasarkan hasil pembahasan dapat disimpulkan bahwa gabungan terpisah graf roda $m W_{n}, n \geq 3$, $m \geq 2$ dan $n \equiv 0 \bmod 3$, memiliki pelabelan total tak teratur total, dengan nilai total ketakteraturan total $t s\left(m W_{n}\right)=\left\lceil\frac{2 m n+2}{3}\right\rceil$. Hal serupa pada gabungan terpisah graf buku segitiga, $\left(m P_{1} \odot S_{n}\right), n \geq 3, n \equiv 1 \bmod 3$, dan $m \geq 1$ dengan nilai total ketakteraturan total $t s\left(m\left(P_{1} \odot S_{n}\right)\right)\left\lceil\frac{m(2 n+1)+2}{3}\right\rceil$.

\section{Daftar Pustaka}

[1] M. Baca, S. Jendrol, M. Miller and J. Ryan, "On Irregular Total Labelings," Discrete Mathematics, vol. 307, pp. 1378-1388, 2007.

[2] K. Wijaya and Slamin, "Total Vertex Irregular Labelings of Wheels, Fan, Suns, and Friendship Graphs," 2008.

[3] Nurdin, A. N. M. Salman and E. T. Baskoro, "The Total Edge Irregular Strength of the Corona Product of Path with Some Graphs," 2008.

[4] J. A. Galian, "A Dynamic Survey of Graph Labeling," Electronic Journal of Combinatorics, vol. 17, no. \#DS6, 2014.

[5] C. C. Marzuki, A. N. M. Salman and M. Miller, "On The Total Irregularity Strength of Cycles and Paths," Far East J. Math. Sci. , vol. 82, no. 1, pp. 1-21, 2013.

[6] R. Ramdani and A. N. M. Salman, "On The Total Irregularity Strength of Some Cartesian Product Graphs," $A K C E$ Int. J. Graphs Comb., vol. 10, pp. 199-209, 2013.

[7] M. I. Tilukay, A. N. M. Salman and E. R. Persulessy, "On The Total Irregularity Strength of Fan, Wheel, Triangular Book, and Friendship Graphs," Procedia Computer Science, vol. 74, pp. 124-131, 2015. 